The data definitely support coloured quarks.

\section{Open Problems}

The fact that all particle interactions are described with the same type of Yang-Mills theory leaves ample ground to suspect that a further embedding of the Standard Theory into a more unified structure is needed. The peculiar absence of gravity in the present scheme is another valid reason.

It must be stressed, however, that there still remain many areas, within the theory itself, which need further theoretical and experimental investigation.

A definitive consequence of the way the electroweak symmetry is broken is the existence of a neutral spinless particle, the Higgs boson, which has escaped detection so far. The reason why it is so difficult to observe is that it couples to the particle mass, i.e. it is only feebly coupled to the light matter in the available beams. The next generation of machines should produce abundantly various types of heavy particles, $\mathrm{W}, \mathrm{Z}$ and perhaps t-quarks, and make the observation of the Higgs boson possible. Until this particle has been definitely observed, we cannot claim to understand where $W$ and $Z$ get their mass from.

Short distance processes, where perturbative $Q C D$ can be applied, are only the tip of an iceberg made by the wealth of data from non-perturbative processes (hadronic spectra, cross-sections etc.). The present, most promising approach to non perturbative $\mathrm{QCD}$ is computer simulation of the theory on a discrete lattice. Aside from practical results, it is hoped that the large efforts being made in this direction will give valid clues for a more direct and analytic approach.

\section{REFERENCES}

1. The Standard Theory is described in many textbooks, see e.g. Lee T.D., Particle Physics and Introduction to Field Theory (Harwood Academic Publ. Chur, London, New York) 1981.

2. Altarelli G., Proceedings of the 23rd International Conference on High Energy Physics, Berkeley July 16-23, 1986; Marciano W. op. cit.

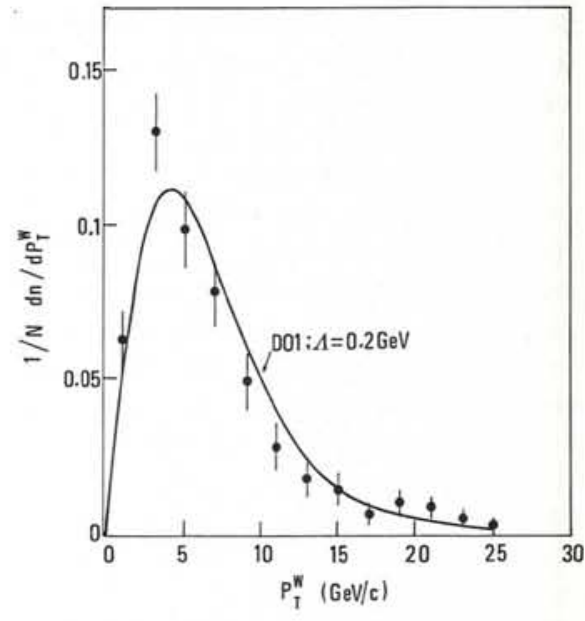

Fig. 1 - Transverse momentum distribution of the $W$ produced in $p-\bar{p}$ collisions at the CERN collider; $P_{T}^{W}$ is the $W$-momentum transverse to the beam. The experimental points are from the UA1 collaboration. The continuous line is the $Q C D$ prediction, normalized to the total number of events, including the experimental resolution and cuts. The theoretical prediction is computed with the structure functions of Duke and Owens, DO1, see ref. 2.

\title{
The Evolution of Particle Accelerators
}

\section{Bryan W. Montague, Geneva}

(CERN)

Over the past forty years or so, particle accelerators have undergone tremendous developments, both in energy and in design subtlety. Each energy region explored has revealed new features of the structure of matter, but has also raised further questions that required higher energies for their elucidation.

Progress in this field has come about both by major conceptual advances and by a steady evolution in theoretical understanding and applied technology. The phase-stability principle and the invention of alternating-gradient focussing had a major and lasting impact, and the concept of colliding beams has become indispensable for elementary-particle physics at the higher energies.

It had been recognised early on that, in collisions between particles of equal energy moving in opposite directions, the combined energy of both particles is available for the reaction. This contrasts with particles incident on a fixed target, where much of the energy goes into moving the centre of mass. However, to exploit the kinematical advantage of colliding beams requires high beam densities in order to obtain adequate interaction rates or, in other words, sufficient luminosity. By the 1960's the understanding of accelerator beam dynamics together with advances in the technology, made it possible to build useful collidingbeam facilities. By this time also their introduction was becoming really necessary because of the size that accelerators had reached.

In the past fifteen years, collidingbeam physics has been pursued in storage rings of roughly circular shape. These machines fall into two main categories, electron-positron colliders and hadron colliders, the latter producing proton-proton (p-p) or proton-antiproton $(p-\bar{p})$ collisions. Both types obtain sufficiently high beam densities by the accumulation of many injected bunches of particles. Production of anti-proton beams of sufficient density became possible thanks to a major innovation, namely stochastic cooling. This relies on progressively applying correction signals derived from average measurements over a cross-section, to a circulating beam in which continuous mixing of the particles occurs. The net result is a reduction in the relativespread as the beam cools.

Future Needs of High-Energy Physics

Accelerators in the future will be colliding-beam machines either for electrons $\left(\mathrm{e}^{+}-\mathrm{e}^{-}\right)$or for hadrons. The difference between these is significant in several respects. First the point-like nature of electrons yields very "clean" physics with relatively little background, and the full energy of the collision is available for producing other particles and states. In contrast, the quark structure of hadrons leads to much of the collision energy appearing in the form of rather uninteresting "soft" interactions, making the "hard" quark-quark collisions less numerous and more difficult to investigate. Consequently, electron colliders are desirable for detailed precision measurements of high-energy processes, whereas hadron colliders have certain advantages for making new discoveries.

For future electron colliders we shall have to abandon the circular storagering shape because of energy loss through synchrotron radiation, which increases with the fourth power of the energy. The Large Electron Positron (LEP) machine under construction at CERN, with a circumference of $27 \mathrm{~km}$ 
and an ultimate energy of $100 \mathrm{GeV}$ per beam, is almost certainly the last of its kind. To reach centre-of-mass energies substantially beyond $200 \mathrm{GeV}$ in $\mathrm{e}^{+}-\mathrm{e}^{-}$ collisions we have to turn to linear colliders, where particles are accelerated along essentially straight trajectories. This introduces some serious practical problems and limitations, which we shall discuss later.

Circular hadron colliders are still a viable option for future high-energy physics, at least for some years to come. Although they require a factor of about ten higher energy as compared with electron colliders of equivalent physics potential, they have negligible synchrotron radiation and their construction involves a less dramatic extrapolation of known techniques. The use of superconducting magnets on a large scale is already under way in the proton ring of the HERA electron-proton collider at Hamburg (Fig. 1). Fields as high as 10 tesla can now be foreseen with confidence, and a Large Hadron Collider (LHC) could be built in the LEP tunnel to provide hadron collisions at around 17 $\mathrm{TeV}$ in the centre-of-mass. In the USA a more ambitious Superconducting Super Collider (SSC) of 20-40 TeV is under study. At even higher energies, saturation of the detectors may make hadron machines unusable, and $\mathrm{e}^{+}-\mathrm{e}^{-}$linear colliders would become indispensable for further progress.

\section{Linear Colliders}

Linear accelerators, or linacs, have been in use for many years; they accelerate bunches of particles by means of radiofrequency fields in resonant cavities or waveguides. Two linacs placed end-to-end can be arranged to produce head-on collisions between electron and positron bunches at an interaction region between the two machines.

Although simple in concept, $\mathrm{e}^{+}-\mathrm{e}^{-}$ linear colliders start off at a basic disad- vantage with respect to storage rings, namely that any two bunches can collide only once rather than undergoing encounters on successive revolutions for several hours. To overcome this limitation we have to increase the time-average density of particles at the collision point by increasing the beam current, by reducing the beam emittance and by focussing the colliding beams down to an extremely small cross-section. High beam currents lead to heavy power demands on the radiofrequency driving system, and the power conversion efficiency becomes a crucial issue. Reducing the emittance requires special damping rings, which decrease the beam size by synchrotron-radiation damping, thus increasing the brightness. Tight focussing has already been widely exploited in storage rings and highly unconventional methods are required to make further substantial gains in luminosity. The problems are compounded by the need to achieve even higher luminosities at higher energies, since the point-like cross-section for electro-weak interactions decreases with the square of the energy.

One approach to the problem of focussing in the interaction region is to make use of the space-charge forces acting between electron and positron bunches. These forces are mutually focussing and, with sufficient bunch intensity, can pinch the beams into a small crosssection within the interaction length. The luminosity can thereby be enhanced by a factor of about five or six, but cannot be pushed any further because of plasma instability. Near the limiting regime the forces are highly non-linear and the bunch quality is degraded. In addition, the beam-beam synchrotron radiation, inelegantly dubbed "beamstrahlung", increases the spread of particle energies in the collision region, introducing uncertainties in the kinematic reconstruction of events.

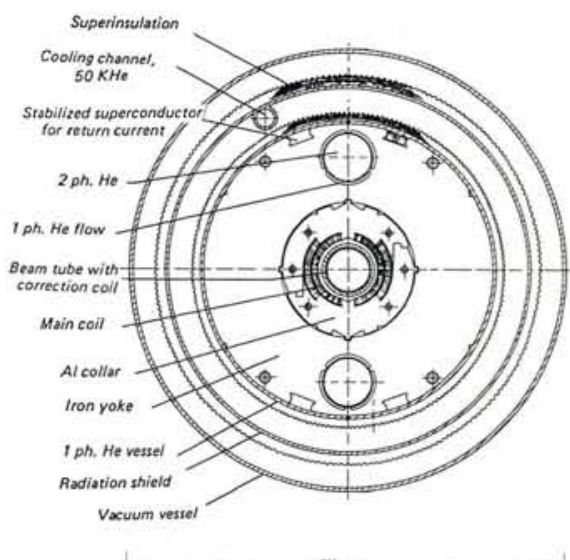

Fig. 1 - Section of the dipole magnet developed at DESY for HERA with very low heat loss. Over $9 \mathrm{~m}$ long they give more than 5T of homogeneous field.

These problems of interaction-region beam optics present a formidable challenge to accelerator designers. However we should not be discouraged, since we are entering a new and unfamiliar field of machine parameters. The theoretical effort now being devoted to these topics, together with the experimental results anticipated when the Stanford Linear Collider (SLC) comes into operation, should resolve many of the problems.

\section{Linac Requirements}

Although the general principles of linear accelerators are very well understood, the requirements of high-energy colliders with high luminosity bring us into a new regime. The detailed interplay of the various factors is very complicated but the more important features can roughly be summarised as follows.

High energy implies high accelerating fields if the linacs are not to be unreasonably long. What used to be a very respectable energy gain of $20 \mathrm{MeV} / \mathrm{m}$ would require $100 \mathrm{~km}$ length for a $1 \mathrm{TeV}$ $+1 \mathrm{TeV}$ collider! Accelerating fields can, however, be pushed up to around 100

\section{Concise Nuclear Isobar Charts}

Nuclear Ground States and Low-Lying Energy Levels

\section{Edited by Hans Bucka}

1986. 5 charts printed in three colours, size $43 \mathrm{~cm} \times 100 \mathrm{~cm}$, folded to fit into portfolio size $32 \mathrm{~cm} \times 44 \mathrm{~cm}$; included is 24 page instruction / introduction manual. DM 78,-; approx. US \$39.00. ISBN 311008404 X

In the Concise Nuclear Isobar Charts, data on binding energies of protons and neutrons in the ground state and excitation energies for lowlying nuclear energy levels are displayed, both of which are of great interest for transition processes as well as for questions of nuclear structure.
Also, quantum numbers for angular momentum and parity are shown for these energy levels. For the stable nuclei, data for the relative abundances, and for unstable nucleon configurations, the transition probabilities are included in the data displayed.

Due to the representation chosen for the atomic nuclei, in many cases a very clear first survey of systematic properties of nuclear energy states as well as spontaneous decay processes is achieved.

Price is subject to change without notice

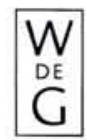

de Gruyter · Berlin · New York 
$\mathrm{MV} / \mathrm{m}$, even at the conventional radiofrequency wavelength of $10 \mathrm{~cm}$, but at the expense of increased power loss. For higher accelerating fields we have to go to shorter RF wavelengths, in order to avoid electrical breakdown in the accelerating cavities. However at wavelengths below about $1 \mathrm{~cm}$ it becomes difficult to construct RF power generators of sufficiently high power and efficiency.

In view of focussing limitations at the interaction region, high luminosity requires high beam currents and therefore high beam power, which has to be provided by the stored energy in the RF accelerating structure. Both fundamental and technological considerations limit the efficiency of this energy transfer, which becomes a crucial practical issue for high-energy linear colliders. In particular, high peak beam currents induce wake fields in the accelerating cavities which degrade the quality of the beam. These wake fields are analogous to the turbulent wake left by a fast boat, and arise from the interaction between the charged beam and conducting boundaries. They are of special concern at the shorter wavelengths, where the cavities are necessarily of small transverse dimensions, which in itself poses problems of fabrication and tolerances.

\section{Solutions for the Future}

Many ideas have been put forward for dealing with the problems of acceleration to very high energies, and several workshops on these topics have been held in Europe and in the USA; the proceedings of these are listed in the Bibliography.

Some of the schemes are based on more or less conventional accelerator structures but for shorter wavelengths. The variants are characterised by the methods used for generating the RF power, which could be by a Free Electron Laser (FEL) or through wake fields induced by a driving beam consisting of very short bunches. Most of these methods are in fact based on the extraction of power from a low-to-medium energy, high-current driving beam, the extracted energy being restored at intervals either by a second, superconducting linac (Fig. 2 ), acting as an energy storage device, or by a pulsed induction linac. A less conventional proposal is to excite the accelerating structure by high-voltage pulses of a few picoseconds duration, produced by photo-diode switches triggered from laser pulses.

The semi-conventional schemes could offer accelerating fields in the range of a few hundred MV/m. A much less conventional approach is to excite accelera-

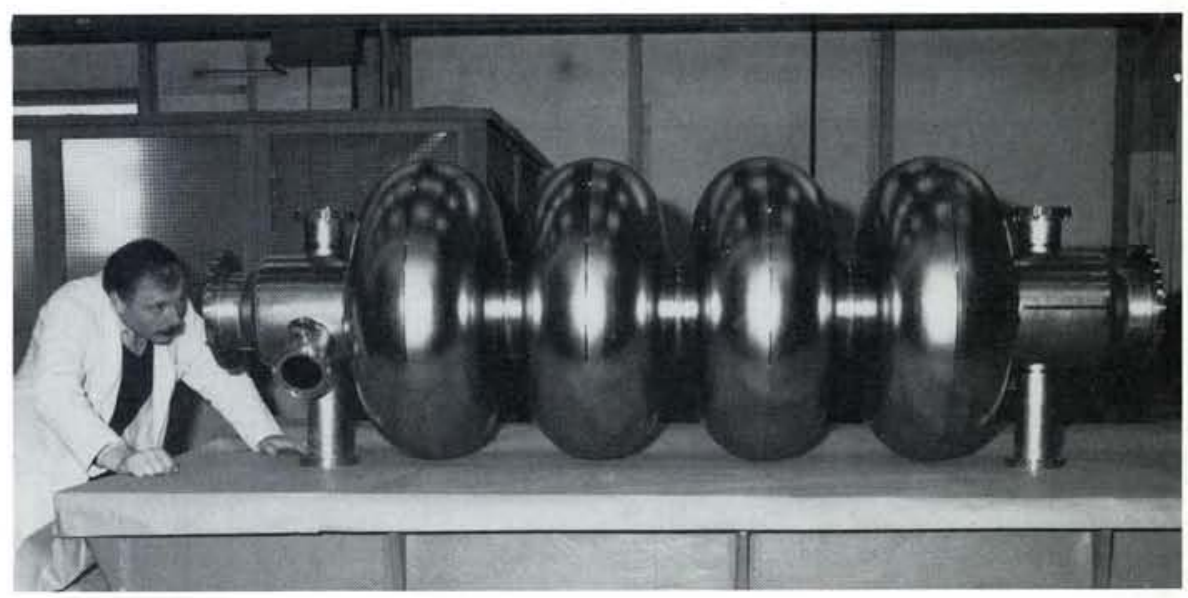

Fig. 2 - Superconducting Nb RF accelerating cavity for the CERN large Electron-Positron Collider (LEP). Such cavities would be directly applicable to energy restoration of the high current driving beam for the CERN Linear Collider (CLIC) currently under study.

ting fields directly in the same structure as the accelerated beam from the wake fields of an intense bunched driving beam. Although such wake-field accelerators might give energy gains up to around $1 \mathrm{GeV} / \mathrm{m}$, there is still some concern about the beam stability in such a tightly-coupled system.

The only means so far proposed to achieve energy gains substantially above $1 \mathrm{GeV} / \mathrm{m}$ involve the use of plasmas. The principle is based on charge separation in a fully-ionised plasma with a density in the range of $10^{16}-10^{18}$ $\mathrm{cm}^{-3}$, which can produce fields of several GV/m. In the Plasma Beat-Wave Accelerator the charge separation is obtained by excitation from two coincident laser beams whose frequency difference resonates with the plasma frequency. The Plasma Wake-Field Accelerator obtains the charge separation from the wake fields of an intense bunched driving beam. Although plasmas have a bad reputation for instabilities, the time scale of interest for accelerator applications is only a few tens of picoseconds which is expected to be too short for instabilities to develop. Nevertheless it will probably be many years before a full-scale project based on plasma waves can be contemplated. On the other hand, the exploitation of these extremely high fields on a smaller scale, for focussing near the interaction region, could perhaps become feasible much earlier.

\section{Conclusions}

Since the early 1950's, the centre-ofmass energy available from accelerators has increased by a factor of over five hundred at an increase in real cost of five to ten. The question now facing the high-energy physics community is whether this evolution can continue in the future, even at a slower pace. Accelerators have now reached a very large size and their cost, though modest on the scale of many other human activities, is rather conspicuous in a social and political environment much less sympathetic to basic science than hitherto. There is thus a particularly strong incentive today to seek even more economical methods of accelerating particles to higher energies.

These facts of life are very well recognised in the high-energy physics community, and many groups, with very limited resources, are investigating the future possibilities with great enthusiasm. High-energy physics is, for well-known fundamental reasons, necessarily a rather expensive basic science. However, it cannot be neglected without a serious long-term risk of leaving other, more tangible, branches of research without roots from which to feed. It takes a generation to build up the expertise of a large research activity, but only very few years of inadequate support to destroy it.

\section{BIBLIOGRAPHY}

1. Laser Acceleration of Particles (Los Alamos, 1982)', ed. P.J. Channell, AIP Conference Proceedings 91.

2. 'The Challenge of Ultra-High Energies', Proc. ECFA-RAL Meeting, Oxford 1982, ECFA 83-68.

3. 'The Generation of High Fields for Particle Acceleration to Very High Energies', Proc. CAS-ECFA-INFN Workshop, Frascati 1984, ECFA 85/91, CERN 85-07.

4. 'Laser Acceleration of Particles (UCLAMalibu, California, 1985)', ed. Chan Joshi and Thomas Katsouleas, AIP Conference Proceedings 130.

5. 'Proc. 1985 Particle Accelerator Conf. (Vancouver 1985)', IEEE Trans. Nucl. Sci. 32, No. 5.

6. 'Symposium on Advanced Accelerator Concepts (Madison, Wisconsin, 1986)', to be published in AIP Conference Proceedings. 